\title{
Laser-induced Chemical Transformation of Free-standing Graphene Oxide Membranes in Liquid and Gas Ammonia Environments
}

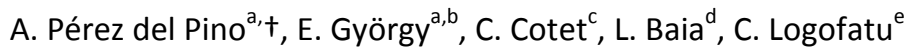

Laser-induced chemical conversion of graphene oxide (GO) is an effective way to modify its properties and expand its potential use for numerous applications. In this work, mechanically stable and flexible free-standing GO membrane is synthesized and further processed by ultraviolet laser radiation in gas and liquid ammonia-rich environments. Electron and atomic force microscopies, as well as X-ray photoelectron spectroscopy analyses reveal that laser irradiation in gas ambient leads to a large defect-induced morphology modification and high deoxygenation process, accompanied by slight incorporation of nitrogen functionalities to the reduced GO structure. Conversely, irradiation in liquid ambient provokes significant integration of nitrogen groups, essentially amines, in a partially reduced GO structure, without evident modification of the morphology. Electrical measurements in the macro- and nano-scale point to a complex contribution of morphology and oxidized regions to the overall resistance of the rGO.

a. Instituto de Ciencia de Materiales de Barcelona, Consejo Superior de Investigaciones Científicas (ICMAB-CSIC), Campus UAB, 08193 Bellaterra, Spain

b. National Institute for Lasers, Plasma and Radiation Physics, P. O. Box MG 36, 77125 Bucharest, Romania.

c. Babes-Bolyai University, Faculty of Chemistry and Chemical Engineering, Department of Chemical Engineering, Arany Janos str.11, RO-400028, Cluj-Napoca, Romania

d. Babes-Bolyai University, Faculty of Physics \& Interdisciplinary Research Institute on Bio-Nano-Sciences, M. Kogalniceanu str. 1, RO-400048, Cluj-Napoca, Romania

e. National Institute for Materials Physics, P. O. Box MG. 7, 77125 Bucharest, Romania

+Corresponding author. Email: aperez@icmab.es. Tel. +34 935801853. FAX. +34 935805729.

\section{Introduction}

Nowadays, the challenge regarding the fabrication of graphenebased materials is closely related to their industrial applications, involving their mass-production preserving the outstanding performances obtained in the research laboratories. ${ }^{1}$ Following this target, low-cost, eco-friendly, and more scalable protocols for the large quantity synthesis of high quality multifunctional graphenebased materials and devices based on them are still a demand. Recently, graphene oxide (GO) nanostructures have attracted great interest due to its exceptional physicochemical properties for many applications. ${ }^{2-6}$. Besides, GO sheets are able to be converted into a graphene-like material, named reduced graphene oxide ( $\mathrm{GGO}$ ), by relatively easy, scalable, and cost-effective synthesis methods. Then, GO arises as a key element for obtaining graphene-like materials at industrial scale.

GO exhibits very interesting functional properties: it is dispersible in water, is biocompatible, and its electrical conductivity and optical band gap can be tailored by just modifying its oxidation degree. Accordingly, GO is an electrical insulator whereas $\mathrm{rGO}$ is more conductive, depending on the deoxygenation level, and can act as a p-type semiconductor. ${ }^{7-9}$ It has been recently reported that $\mathrm{GO}$ and partially reduced $\mathrm{GO}(\mathrm{rGO})$ have tremendous potential to be used in varied electrochemical applications such as photocatalysts due to the complex interplay of the incorporated functional groups and graphitic regions with different external molecules through covalent and noncovalent interactions. ${ }^{3,10,11}$ Furthermore, the functionalization of $\mathrm{rGO}$ with nitrogen species confers additional, $\mathrm{n}$ type nature leading to the formation of a semiconductor with both $\mathrm{p}$ - and n-type characteristics. ${ }^{12,13}$ The resulting synergic combination of mixed $p-n$ heterojunctions in the same material, and the benefits of relatively easy synthesis of $\mathrm{N}$-doped graphenelike films from GO powder, significantly extend the room for the development of new performant rGO-based devices such as nonvolatile memories, transparent electrodes, supercapacitors and photocatalysts. $^{14-17}$

The structural modification of $\mathrm{GO}$ for the synthesis of $\mathrm{rGO}$ and $\mathrm{N}$ doped $\mathrm{rGO}$ can be carried out by chemical and thermal treatments. ${ }^{18}$ However, these methods need of either toxic or high temperature processes which hold up the large scale development of GO and rGO-based devices. Conversely, laser processing arises as a very promising tool for local, harmless and large-scale modification of GO structure. ${ }^{19}$ For instance, laser direct writing has been demonstrated to be a fast and versatile method for the development of patterned rGO-based materials and devices composed of them. ${ }^{20,21}$ Besides, functional rGO-based hybrid composites' thin films can be also processed through this technique onto almost any type of substrate. ${ }^{22,23}$ Furthermore, laser irradiation can generate out-of-thermodynamic equilibrium photochemical and photothermal mechanisms, ${ }^{24}$ leading to the 
generation of new different chemical pathways as compared to conventional methods. Therefore, laser processing methods are certainly opening very promising opportunities for the scalable fabrication of rGO-based devices.

In this work, a simple method is developed for the fabrication of mechanically stable, flexible, and free-standing membranes composed of GO. This method allows obtaining films with areas up to hundreds of $\mathrm{cm}^{2}$. After that, the membranes are treated with nanosecond pulsed ultraviolet laser radiation in gaseous and liquid ammonia-rich ambient. The structural and compositional characterization of the obtained materials reveals significant differences regarding the morphology and chemical composition of samples fabricated under analogous laser conditions but in different environment. These dissimilarities lead to distinct macroscopic and microscopic electrical properties.

\section{Experimental}

GO films of about $20 \mu \mathrm{m}$ in thickness were prepared by a synthesis pathway consisting of (i) three-step graphite oxidation-exfoliation procedure using the main precursors of Marcano-Tour's improved method, ${ }^{25}$ (ii) obtaining the GO solid-precursor by separation in a water-ethanol GO suspension, and (iii) drop-casting of the prepared aqueous $\mathrm{GO}$ suspension on a glass wafer, followed by drying and peeling of the film. In the first step of graphite oxidation-exfoliation method, $270 \mathrm{~mL}$ of $\mathrm{H}_{2} \mathrm{SO}_{4}$ (95-97\% purity), $30 \mathrm{~mL}$ of $\mathrm{H}_{3} \mathrm{PO}_{4}$ (85\% purity) and $2.7 \mathrm{~g}$ of graphite (purum powder, $\leq 0.1 \mathrm{~mm}$, Fulka) were mixed under stirring in an $800 \mathrm{~mL}$ glass jar placed in ice bath. After about $20 \mathrm{~min}, 12 \mathrm{~g}$ of $\mathrm{KMnO}_{4}$ (99\% purity, Merck) was gradually added maintaining the stirring conditions at about $0 \circ \mathrm{C}$ for $2 \mathrm{~h}$. Afterwards, the mixture was held without stirring at room temperature for 4 days. In the second step, the mixture was put again under stirring in ice bath and after $20 \mathrm{~min} 200 \mathrm{~mL}$ of $\mathrm{H}_{2} \mathrm{O}_{2}(3 \%$ vol.) was gradually added (in about $20 \mathrm{~min}$ ). After $1 \mathrm{~h}$, the obtained mixture was centrifuged (5000 rpm for $15 \mathrm{~min}$ ), and the supernatant was decanted away. The remaining solid material was then washed successively with $200 \mathrm{~mL}$ of $\mathrm{H}_{2} \mathrm{O}$ (bidistilled), $100 \mathrm{~mL}$ of $\mathrm{HCl}(37 \%$ vol.) and $100 \mathrm{~mL}$ of absolute $\mathrm{EtOH}$. The last two washings were repeated two times. After each washing the suspension was dispersed by magnetically stirring $(5 \mathrm{~min})$, sonicated (15 min) and centrifuged (5000 rpm for $15 \mathrm{~min}$ ) and the supernatant was decanted away. In the third step, the resulted solid was dispersed and sonicated ( $15 \mathrm{~min}$ ) again in $200 \mathrm{~mL}$ of a $50 \%$ vol. aqueous ethanol solution. The resulted $\mathrm{GO}$ suspension was held in a closed jar for 7 days in order to produce the separation by sedimentation: the expanded graphite remaining after oxidation as well as the macroscopic graphite oxide precipitated. It is known that GO sheets form very stable dispersions in water because of their strong hydrophilic character and sediments in pure ethanol. ${ }^{26}$ Thus, on the top side of the jar only the water-ethanol suspension with floating GO nanosheets was formed. Afterwards, about $200 \mathrm{~mL}$ of this initial GO suspension was harvested from the above part of the jar and dried in open atmosphere for 3 days. A dark-brown solid GO was obtained. Then, a $2.5 \mathrm{mg} \mathrm{mL}^{-1}$ of $\mathrm{GO}$ suspension was obtained by dispersing solid $\mathrm{GO}$ in bidistilled water by magnetically stirring. This suspension was then poured on a smooth glass wafer and dried for 3 days in ambient conditions. The resulted GO film was peeled off with the aid of a wide blade. This method allows obtaining freestanding, mechanically stable and flexible GO membranes of hundreds of $\mathrm{cm}^{2}$ dimensions in a straightforwardly way.
GO membranes were cut in pieces of few $\mathrm{cm}^{2}$ (Fig. 1a) and submitted to laser treatment at room temperature. Irradiation experiments were made using a $266 \mathrm{~nm}$ wavelength Quantel Brilliant Nd:YAG laser system, emitting pulses with 3 ns duration and $10 \mathrm{~Hz}$ repetition rate. Irradiations were performed by means of a squared laser spot, $1 \times 1 \mathrm{~mm}^{2}$ in size, and applying subsequent laser pulses in the 1-1000 range. The incident laser fluence values were fixed at 50 and $100 \mathrm{~mJ} \mathrm{~cm}^{-2}$. Patterns of several $\mathrm{mm}^{2}$ dimensions were obtained by irradiation of adjacent spots with $50 \%$ overlapping (example of pattern at inset in Fig. 1a). Two sets of experiments were performed in ammonia $\left(\mathrm{NH}_{3}\right)$-rich reactive environments. In the first set of experiments, $\mathrm{GO}$ membranes were irradiated inside a reaction chamber previously evacuated down to a residual pressure of $10^{-4} \mathrm{~Pa}$. The laser treatments were performed in gaseous ambient by flowing $\mathrm{N}_{2}$ gas through a $\mathrm{NH}_{3}-\mathrm{H}_{2} \mathrm{O}$ liquid solution $(30 \% \mathrm{v} / \mathrm{v})$ and introducing the gas mixture in the reaction chamber at nearby atmospheric pressure. In the second set of experiments, $\mathrm{GO}$ membranes were directly immersed in $\mathrm{NH}_{3}-\mathrm{H}_{2} \mathrm{O}$ liquid solution $(30 \% \mathrm{v} / \mathrm{v})$, inside a quartz vessel. The laser treatments were performed using the same irradiation conditions in gas and liquid ambient. For convenient comparisons, the raw GO membrane and the most significant laser treated samples were coded and summarized in Table 1.

\begin{tabular}{|l|l|}
\hline Code & Sample \\
\hline Ref & As synthesized GO film. \\
\hline G50 & $\begin{array}{l}\text { GO irradiated in gaseous } \mathrm{NH}_{3} \text {-rich atmosphere. } 50 \mathrm{~mJ} \\
\mathrm{~cm}^{-2}, 500 \text { pulses/zone. }\end{array}$ \\
\hline G100 & $\begin{array}{l}\text { GO irradiated in gaseous } \mathrm{NH}_{3} \text {-rich atmosphere. } 100 \mathrm{~mJ} \\
\mathrm{Cm}^{-2}, 500 \text { pulses/zone. }\end{array}$ \\
\hline LRef & $\begin{array}{l}\mathrm{GO} \text { membrane exposed to liquid } \mathrm{NH}_{3} \text {-rich ambient for } \\
\text { ca. } 1 \mathrm{~h} \text { (not irradiated). }\end{array}$ \\
\hline L100 & $\begin{array}{l}\text { GO irradiated in liquid } \mathrm{NH}_{3} \text {-rich ambient. } 100 \mathrm{~mJ} \mathrm{~cm}^{-2}, \\
500 \text { pulses/zone. }\end{array}$ \\
\hline
\end{tabular}

Table 1. Sample identification as a function of experimental conditions.

The morphology of the as synthesized as well as irradiated layers was studied by field emission scanning electron microscopy (SEM) with a FEI QUANTA 200 FEG-ESEM equipment, and atomic force microscopy (AFM) through an Agilent 5100 system working at intermittent contact mode. X-ray photoelectron spectroscopy (XPS) was used for the surface compositional study of the obtained materials. The measurements were done in $10^{-7} \mathrm{~Pa}$ ultra-high vacuum ambient by means of a SPECS XPS spectrometer based on Phoibos 150 electron energy analyzer. The system operated in constant energy mode. A monochromatic Al Ka line (1486.61 eV) Xray source was used for excitation. High resolution spectra were recorded over $20 \mathrm{eV}$ range with $10 \mathrm{eV}$ pass energy and $0.7 \mathrm{eV}$ energy resolution. The electrical nature of the processed layers was studied by means of van der Pauw resistance measurements in $5 \times 5$ $\mathrm{mm}^{2}$ samples using a Keithley $2612 \mathrm{~A}$ source-meter system. Furthermore, resistance maps at the microscale as well as currentvoltage spectroscopies at nanometric locations were acquired with an Agilent 5500LS scanning probe microscope equipped with a Resiscope II module (CSI). The measurements were obtained using 
platinum-coated silicon tips (RMN-25-PT300, Rocky Mountain Nanotechnology) and in less than $10 \%$ relative humidity ambient to avoid undesired electrochemical reactions. The bias voltage is applied to the samples surface, where silver paste contacts are connected. The AFM and resistance maps data were processed with Mountains 7.2 software from Digital Surf. Finally, approximate numerical calculations were made in order to get understanding of the thermophysical mechanisms appearing during the irradiation of GO material. For the sake of simplicity, photochemical mechanisms were not considered and only photothermal processes were simulated in 2D models by means of COMSOL 5.2 multiphysics software. A description of the model can be found in Refs. [22,27]. The time evolution of temperature of $20 \mu \mathrm{m}$ thick GO membranes in contact with air (gas) and water (liquid) during irradiation with one laser pulse at 50 and $100 \mathrm{~mJ} \mathrm{~cm}^{-2}$ was assessed. The simulation included the coupling of heat transfer and turbulent fluid dynamics models. Graphene oxide thermal and optical parameters were identical to those used in ref. [22], whereas the thermophysical and mechanical-dynamical properties of the fluids were provided by COMSOL materials library.

\section{Results and discussion}

Raw GO membranes show dark brown-grey color and smooth surfaces under visual inspection. Nevertheless, SEM inspection reveals irregular topography with hundreds of $\mathrm{nm}$-sized protrusions and ripples, caused by bending of $\mathrm{GO}$ sheets during their stackingdrying process (Fig. 1b). The laser irradiation of GO membranes in gaseous ammonia-rich ambient leads to a clear modification of the surface morphology after the accumulation of 100 laser pulses. In Fig. 1c, mountain-like structures with a few microns in size can be appreciated. These features show round aspect, pointing to melting and merging mechanisms of the irradiated material as the mean cause of their formation. Furthermore, high resolution AFM images show that most of the mountain-shaped structures obtained with $100 \mathrm{~mJ} \mathrm{~cm}^{-2}$ laser fluence present terrace- and small filament-like features around 50-100 nm in height-width (Figs. 2a and b). These features tend to align forming parallel bundles, and can reach over few micron areas. The extent and size of the bundles increase with the number of accumulated pulses. However, they are less developed at $50 \mathrm{~mJ} \mathrm{~cm}^{-2}$ laser fluence. Besides, no filamentous features are observed in samples obtained with this laser fluence. Laser irradiation of GO membrane immersed in liquid ammonia solution leads to a small modification of the film color, being somewhat pale grey. However, SEM and AFM studies reveal that even after accumulation of 500 laser pulses per zone at $100 \mathrm{~mJ} \mathrm{~cm}^{-2}$ fluence no significant alteration of the surface morphology is provoked (Figs. $1 \mathrm{~d}$ and $2 \mathrm{c}$ ). Thus, samples irradiated in liquid media are similar to raw GO membrane.

According to our photothermal numerical simulations the absorbed laser energy of each pulse induces fast thermal cycles up to about 1 $\mu \mathrm{s}$ in duration in GO material (Fig. 3). Heating times are very short, in the ns range, with temperature raising rates reaching up to $10^{12} \mathrm{~K}$ $\mathrm{s}^{-1}$,
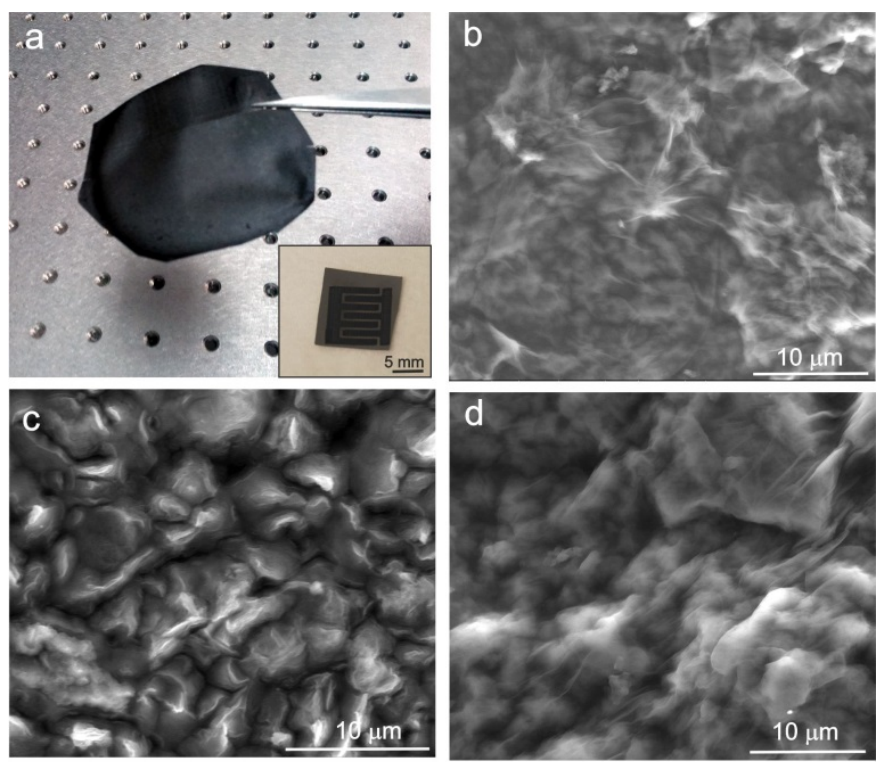

Fig 1. (a) Image of a GO membrane piece. Inset: interdigital pattern processed on GO membrane in ammonia-rich gaseous ambient. SEM images of (b) raw GO membrane, (c) G100 and (d) L100 samples.

cooling times are orders of magnitude longer. As it can be observed, an increase of the applied laser fluence leads to an augment of the maximum temperature as well as the thermal cycle duration. Moreover, the high temperature region is confined to just a few hundreds of nanometers in depth (inset of Fig. 3). Interestingly, and in accordance with the experimental results, the effect of laser radiation on temperature evolution is larger in samples irradiated in gaseous environment than in liquids. Thus, while the maximum temperature reaches up to $3000 \mathrm{~K}$ in GO irradiated in gas at $100 \mathrm{~mJ}$ $\mathrm{cm}^{-2}$, it only reaches $1400 \mathrm{~K}$ when irradiated in similar laser conditions but in liquid medium. This difference in thermal response is due to the larger thermal energy transferred from the heated GO membrane to the liquid surrounding medium as compared to the gaseous one. It should be mentioned that, according to our numerical simulations, the velocity magnitude of the liquid in contact with the GO membrane has negligible velocity up to several microseconds after the starting of the heating process. Thus, convection movements of the liquid would not significantly influence the GO thermal response.

Ultraviolet radiation pulses are expected to cause photochemical transformation of the material's structure as well as rapid temperature variations. These mechanisms, with their respective role, would be at the origin of the observed morphology evolution in samples irradiated in gaseous ambient. Our numerical calculations show that the irradiated material does not reach its melting temperature (if considered to be about $4800 \mathrm{~K}$, as graphene backbone) under single pulse irradiation conditions. Indeed, SEM micrographs of the irradiated samples do not show considerable morphological changes till 100 accumulated pulses. Conversely, at this number of pulses, molten-like structures can be distinguished 

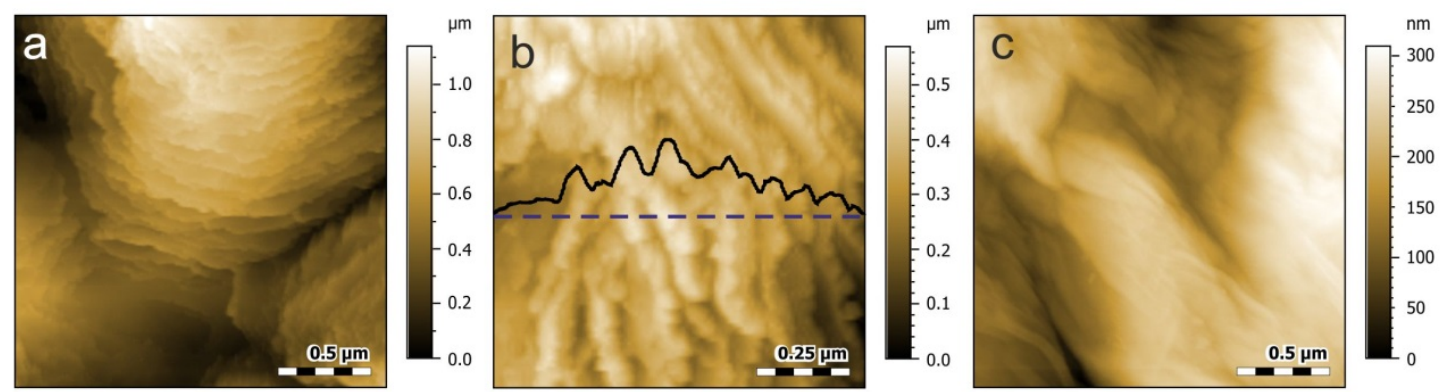

Fig. 2. AFM topographic maps of (a, b) G100, and (c) L100 samples. Inset in b: topographic profile along the dashed line.

(Fig. 1c). Filament-like structures point to the laser-induced creation of structural defects in GO material which provoke a steady stressinduced bending of the GO sheets. ${ }^{28-30}$ The observed terrace-like features would denote the edges of stacked GO sheets. Upon the accumulation of pulses, the proliferation and propagation of crystalline defects would take place. Then, the sites with sufficient density of crystalline defects, exhibiting chemical bonds with lower dissociation energy, would behave as nucleation sites for local premelting and even vaporization processes at temperatures well below the melting point of graphene. ${ }^{31}$ Therefore, the accumulation of laser pulses would promote the concomitant melting to some depth of extended regions of the graphene-based material, as observed in samples processed with high number of accumulated pulses below the melting laser fluence threshold. This mechanism, which is common to the laser processing of many types of materials, is the so-called "incubation effect" ${ }^{24,27,32}$ Conversely, as mentioned, GO membranes irradiated in liquid environment do not exhibit any apparent morphological modification. The causes could be directly linked to the nature and amount of photochemicallyinduced structural defects, as will be presented thereafter, and by the lower temperature development in the material, as revealed by our numerical simulations.

The observed laser-induced morphology evolution is closely related to the compositional modification of the GO material. X-ray photoelectron spectroscopy is an excellent tool for analyzing the chemical structure evolution at the GO's surface. Atomic concentration of $\mathrm{C}, \mathrm{O}$, and $\mathrm{N}$ elements, calculated from the wide scan XPS spectra, are shown for raw GO membrane (Ref), samples irradiated in gaseous (G50, G100) and liquid (L100) environments, as well as GO membrane immersed in liquid solution for about 1 hour (LRef) without laser treatment (Fig. 4a). The atomic concentration of $\mathrm{C}, \mathrm{O}$, and $\mathrm{N}$ elements in as synthesized $\mathrm{GO}$ membrane (Ref) is 70.7, 28.4, and $0.9 \%$, respectively. Laser irradiation of GO membrane in gaseous ammonia-rich atmosphere provokes a high reduction process since $\mathrm{C}$ concentration increases to 91.8 and $91.7 \%$ for G50 and G100, respectively; whereas oxygen

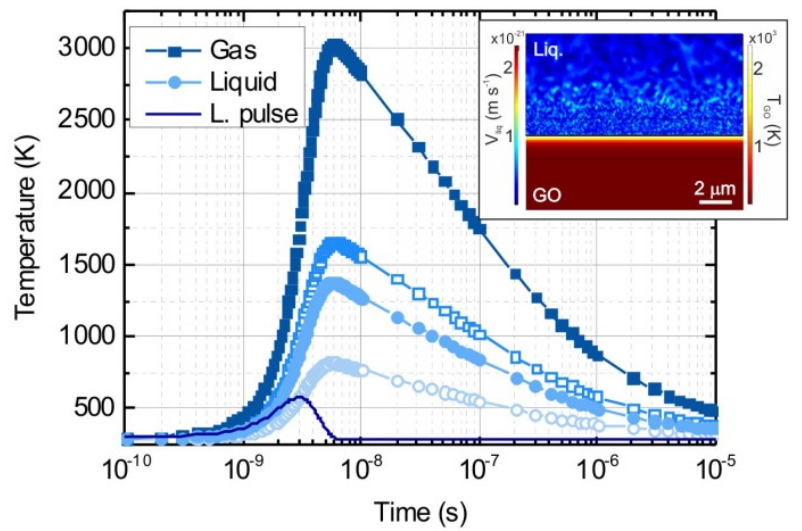

Fig. 3. Simulated temperature evolution with time caused by one laser pulse. Square and circle symbols refer to irradiation in gaseous and liquid ambient, respectively. Open and solid symbols respectively represent irradiation with 50 and $100 \mathrm{~mJ} \mathrm{~cm}^{-2}$ laser fluence. Laser pulse intensity time evolution, in arbitrary units, is also plotted for reference. Inset: temperature in GO membrane and velocity magnitude of liquid water distributions at $6 \mathrm{~ns}$, irradiated with $100 \mathrm{~mJ} \mathrm{~cm}^{-2}$ laser pulse.

concentration abruptly decreases to 7.0 and $6.8 \%$, respectively. Nitrogen is incorporated to the rGO structure in some extent since its concentration slightly increases to $1.2 \%$ (G50) and $1.5 \%$ (G100). We recall that $\mathrm{G} 50$ and $\mathrm{G} 100$ are obtained by accumulation of the same number of laser pulses. Therefore, doubling of laser fluence, despite the large difference in thermal behavior, provokes a rather small difference in $\mathrm{GO}$ reduction and nitrogen doping. The evolution of GO membranes' chemical composition under irradiation in liquid environment is different. First of all, the non-irradiated GO 


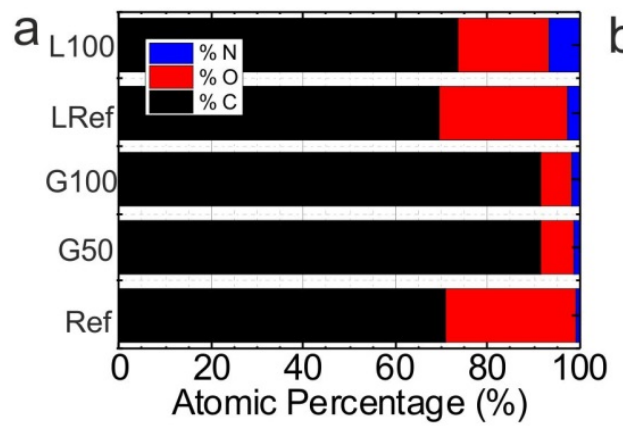

C

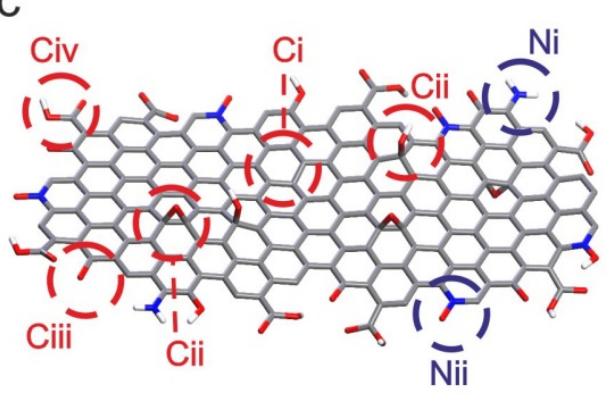

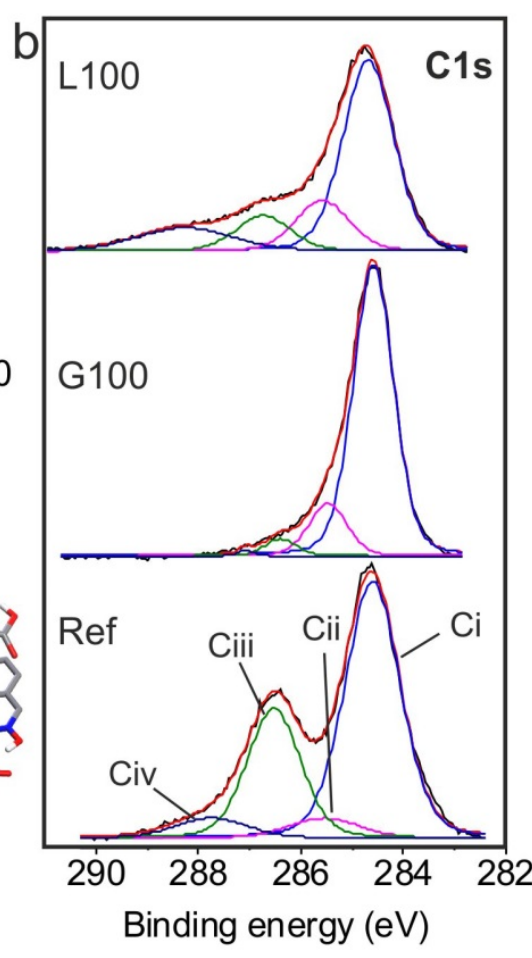

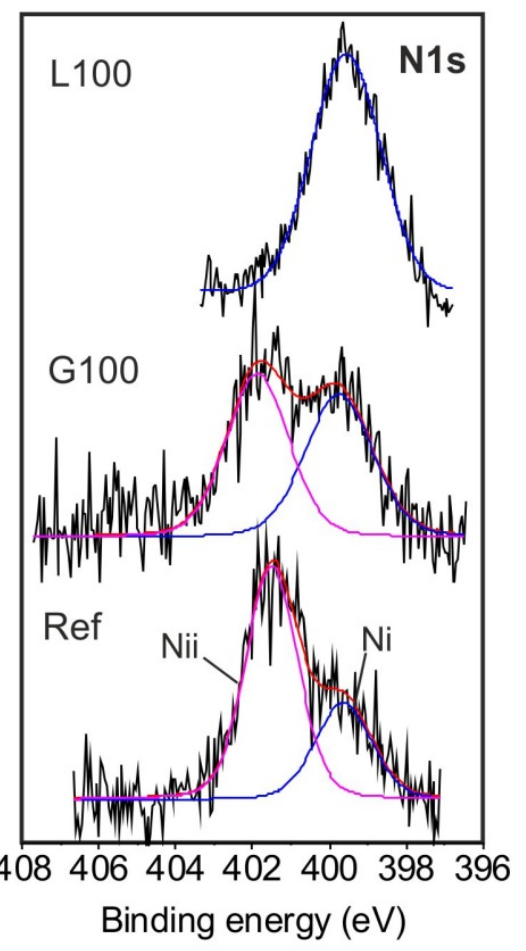

Fig. 4. (a) XPS atomic concentration of $C, O$, and $N$ species; (b) C1s and N1s XPS spectra of different samples. (c) Scheme, according to the Lerf-Klinowski model, of the observed chemical groups in $\mathrm{rGO}$ structure.

membrane immersed in the solution (LRef) exhibits a slight decrease of $\mathrm{C}$ and $\mathrm{O}$ concentration to $69.4 \%$ and $27.8 \%$, respectively. But more interestingly, the $\mathrm{N}$ content noticeably increases up to $2.8 \%$. Laser irradiation at $100 \mathrm{~mJ} \mathrm{~cm}^{-2}$ fluence (L100) leads to a rather limited reduction of $\mathrm{GO}$ material, given that $\mathrm{C}$ concentration slightly increases to $73.8 \%$ and oxygen content moderately decreases to $19.8 \%$. Conversely, $\mathrm{N}$ concentration markedly increases up to $6.4 \%$, that is more than four-fold the concentration reached after similar irradiation process in gaseous ambient. High resolution C1s XPS spectra were recorded and deconvoluted in four peak contributions centered at $284.6 \mathrm{eV}$ (Ci), $285.6 \mathrm{eV}$ (Cii), $286.5 \mathrm{eV}$ (Ciii) and $287.8 \mathrm{eV}$ (Civ) (Fig. 4b). (Ci) is attributed to graphitic carbon $\mathrm{sp}^{2}$ bonds $(\mathrm{C}=\mathrm{C})$, whereas the rest of contributions are respectively assigned to carbon-oxygen (Cii) epoxide-hydroxyl ( $\mathrm{C}-\mathrm{O}-\mathrm{C}, \mathrm{C}-\mathrm{OH}$ ), (Ciii) carbonyl ( $\mathrm{C}=\mathrm{O})$, and (Civ) carboxyl (O-C-OH) bonds (Fig. 4c). ${ }^{22}$ As observed, $\mathrm{Ci}$ and Ciii signals are the main contributions in raw GO membrane (Ref) spectrum. The spectrum of $\mathrm{G} 100$ sample reveals an intense $\mathrm{Ci}$ signal, whereas those assigned to carbon-oxygen bonds show large intensity shrinkage, and Civ signal is even not present. This result, which corroborates the elemental concentration results (Fig. 4a), certainly points to a high degree of deoxygenation of GO material. Conversely, and as expected from element concentration data, the spectrum of L100 sample reveals more intense carbon-oxygen signal than G100, being Cii contribution larger than Ciii and Civ. Deconvoluted N1s XPS spectrum of Ref sample is characterized by the presence of two contributions: (Ni) centered at $400.0 \mathrm{eV}$ and (Nii) centered at $402.0 \mathrm{eV}$. These signals are respectively attributed to amine and pyridinic $\mathrm{N}^{+}-\mathrm{O}^{-}$chemical groups (Fig. 4c). ${ }^{22}$ In this sample, Nii signal is larger than $\mathrm{Ni}$ one, indicating that the small quantity of nitrogen present in raw GO membrane is predominantly due to pyridinic $\mathrm{N}^{+}-\mathrm{O}^{-}$groups created during the synthesis of $\mathrm{GO}$ sheets. Conversely, the spectrum of $\mathrm{G} 100$ reveals that $\mathrm{Ni}$ signal increases almost reaching the level of $\mathrm{Nii}$ one. Then, additional nitrogen is indeed included in the rGO structure during laser irradiation mainly as amine functionalities. This tendency is completely validated in laser irradiation of GO membrane in liquid medium, since N1s spectrum of L100 sample reveals a single and more intense $\mathrm{Ni}$ (amine) signal. It has to be noted that the presence of C-N bonds would contribute in some extent to Civ signal. ${ }^{14}$

In summary, as synthesized GO membrane structure is mainly composed of graphene sheets highly decorated with carbonyl, epoxide, hydroxyl and carboxyl chemical groups, in addition to a tiny amount of pyridinic $\mathrm{N}^{+}-\mathrm{O}^{-}$and amine moieties. Upon laser irradiation in gaseous $\mathrm{NH}_{3}-\mathrm{H}_{2} \mathrm{O}-\mathrm{N}_{2}$ ambient, a prominent removal of oxide-based functional groups is obtained, especially doubled bonded carbon-oxygen ones, besides a slight incorporation of nitrogen-based functionalities in form of amine groups. It is known that $\mathrm{sp}^{2} \pi$-conjugated domains present in $\mathrm{GO}$ confers to it semiconducting properties with a band gap in the $2.5-4.0 \mathrm{eV}$ range, which is determined by the extent of oxygenation of the graphene backbone. ${ }^{5,33-37}$ In our experiments $4.7 \mathrm{eV}$ photons are used for irradiation, exceeding this band gap energy range. Then, photoexcitation of $\mathrm{sp}^{2}$ domains would take place resulting in the generation of electron-hole pairs which would react with GO functional groups and ambient molecules. The presence of water is crucial in the reduction process of GO by means of the following reactions:

$$
\begin{aligned}
& \mathrm{GO}+\mathrm{hv} \rightarrow \mathrm{GO}\left(\mathrm{h}^{+}+\mathrm{e}^{-}\right) \\
& 4 \mathrm{~h}^{+}+2 \mathrm{H}_{2} \mathrm{O} \rightarrow \mathrm{O}_{2}+4 \mathrm{H}^{+} \\
& \mathrm{GO}+4 \mathrm{e}^{-}+4 \mathrm{H}^{+} \rightarrow \mathrm{rGO}+2 \mathrm{H}_{2} \mathrm{O}
\end{aligned}
$$


Matsumoto et al. propose that $\mathrm{H}_{2} \mathrm{O}$ molecules firstly react with epoxy and hydroxyl groups, followed by the rest of oxide functional groups, during UV photoreduction of $\mathrm{GO}$ in the presence of $\mathrm{H}_{2} \mathrm{O}$, leading to the formation of structural defects in the graphene's basal plane. ${ }^{35}$ These reactions would combine carbon atoms from the graphene's structure with oxygen producing $\mathrm{CO}_{2}$ as byproduct, leaving a high density of holes and edges in rGO sheets. These statements would support the relation between large deoxygenation process, the formation of large quantities of structural defects, and the observed morphology variation in G100 sample. However, G100 and L100 XPS spectra clearly show that, in our case, doubled bonded carbon-oxygen groups are mainly removed probably due to the cross-chemical activity of $\mathrm{NH}_{3}$ molecules. ${ }^{17}$ Additionally, it must be pointed out that, given the much higher density of $\mathrm{NH}_{3}$ and $\mathrm{H}_{2} \mathrm{O}$ molecules in liquid ambient than in the gaseous one, higher photochemical reactivity would be expected in the former case. Nevertheless, the obtained degree of deoxygenation and laser-induced variation of morphology in GO processed in gas is much larger than in liquid solution (Figs. 1, 2 and 4). Taking into account that numerical simulations point to the development of much larger temperatures in samples irradiated in gaseous environment than in liquid (Fig. 3 ), it could be suggested a greater impact of photothermal rather than photochemical mechanisms in the reduction of GO and associated formation of structural defects and morphology changes. Indeed, the thermal reduction of $\mathrm{GO}$ is reported to take place in rather low temperature range, i. e. $400-1300 \mathrm{~K}^{38,39}$ Laser treatment of GO membrane in liquid solution actually reaches ca. $1400 \mathrm{~K}$, leading to thermal reduction of $\mathrm{GO}$, but no remarkable morphological change is observed. Then, the noticeable distortion of GO surface as well as formation of micrometric mountain-like features observed in samples obtained in gas ambient could be mainly ascribed to high reduction, dense defect creation and large mechanical stress developed by much extensive thermal processes. ${ }^{36}$ It is worth noticing that GO material with high density of structural defects is preferred for catalytic applications since structural defects on the graphene sheets could lead to stronger catalyst-support interactions thus contributing to an enhanced catalytic performance. ${ }^{35,40}$ As for the nitrogen functionalities, initial pyridinic $\mathrm{N}^{+}-\mathrm{O}^{-}$(Nii) and amine (Ni) groups present in raw GO membrane evolve to higher amount of amine ones, especially in samples irradiated in liquid solution, where significant formation of amine functionality takes place. Recent works point to the formation of amine groups in $\mathrm{GO}$ structure when submitted to low temperature treatments in ammonia ambient ${ }^{41}$ supporting our results. It must be also noted that partially reduced GO containing amine species would exhibit photocatalytic activity with improved efficiency in overall water decomposition into $\mathrm{H}_{2}$ and $\mathrm{O}_{2}$ at visible wavelengths. ${ }^{17}$ Therefore, the presented laser processing method could be a simple and versatile way to obtain photoactive materials for catalytic applications.

The laser-induced modification of the morphology and composition of GO membranes are expected to highly influence its functional properties, such as electrical conductivity. Fig. 5a shows the macroscopic resistance measured by van der Pauw method in $5 \times 5$ $\mathrm{mm}^{2}$ samples. Accordingly, raw GO membrane (Ref) displays a high sheet resistance (ca. $1 \mathrm{M} \Omega / \mathrm{sq}$ ) due to its high oxygenation degree. Furthermore, laser irradiation of GO in gaseous environment (G100) leads to the reduction of the sheet resistance to around $1.1 \mathrm{k} \Omega / \mathrm{sq}$, whereas analogous treatment in liquid (L100) provokes a decrease of resistance to about $54 \mathrm{k} \Omega / \mathrm{sq}$. Many reported works demonstrate that the charge propagation in GO-based materials mainly occurs over $\mathrm{sp}^{2}$ conducting pathways. In particular, the charge transport would take place via variable range hopping between graphitic regions separated by $\mathrm{sp}^{3}$ functional clusters. ${ }^{9,42}$ Accordingly, the observed reduction of resistance in GO material after laser irradiation could be ascribed to the propagation and merging of the $\mathrm{sp}^{2}$ domains, which create percolating pathways for the charge flow across the $\mathrm{rGO}$ layers. ${ }^{42}$ XPS results indeed support the direct correlation between amount of oxygenated functionalities and macroscopic resistance. G100 sample, obtained by laser irradiation in gaseous ambient, is the most deoxygenated one and simultaneously exhibits the lowest sheet resistance, while L100 sample, obtained in liquid ambient, presents an intermediate amount of oxygenated functional groups and sheet resistance as compared to Ref and G100. The influence of nitrogen-based functional groups in the overall resistance of $\mathrm{rGO}$ is not clearly discernible, though seems to not be substantial. Besides the chemical composition, the influence of the structural defects in the electrical conductivity of $\mathrm{rGO}$ is an important matter. It is expected that structural defects contained in $\mathrm{sp}^{2}$-conjugated domains, which provoke the observed morphological changes, induce electron scattering mechanisms leading to the increase of electrical resistance. ${ }^{43}$ In order to go beyond in the study of the correlation between structural defects and conduction paths, SPM-based electric characterization was carried out. Local current-voltage spectroscopic measurements (IVs) were acquired in $[-1,1]$ voltage range at several sites of raw GO membrane (Ref), G100, and L100 samples' surface. Typical IVs of the samples are shown in Fig. 5b. IVs taken in Ref sample exhibit a very resistive nature, with surface-tip flowing current at $1 \mathrm{~V}$ bias in the range of 0.1-10 pA. Higher conductivity at negative voltages is acquired, pointing to a Schottky rectifying behavior in the SPM tip-surface junction given the p-type nature of partially reduced GO. ${ }^{8}$ To the contrary, IVs obtained in G100 and L100 samples reveal symmetric behavior between forward and reverse biasing, as well as linear relationship between current and applied voltage in the studied range. These characteristics disclose ohmic nature in the material besides absence of rectification behavior in the contact region between sample's surface and Pt tip. The current ranges at $1 \mathrm{~V}$ biasing are about $100 \mu \mathrm{A}$ and $1 \mathrm{nA}$ for G100 and L100 samples, respectively. Fig. $5 \mathrm{c}$ presents resistance maps obtained by applying $0.5 \mathrm{~V}$ between samples' surface and SPM tip. As observed, the resistance maps taken in Ref sample are very uniform and do not show any characteristic feature. The measured average resistance value is 

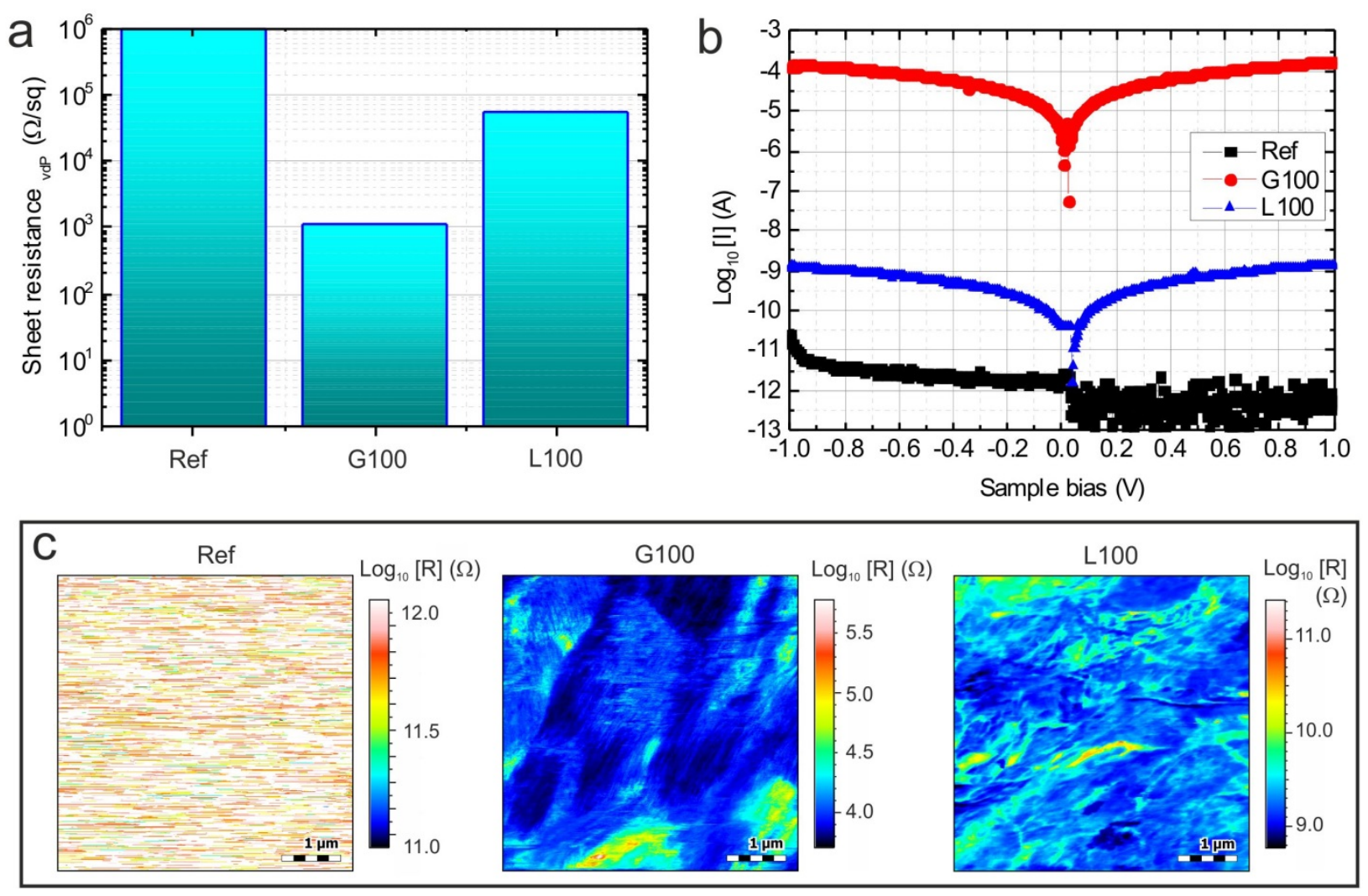

Fig. 5. (a) Macroscopic van der Pauw resistance measurements, (b) local current-voltage spectroscopies, and (c) resistance maps of Ref, G100 and L100 samples. Resistance maps were obtained at $0.5 \mathrm{~V}$.
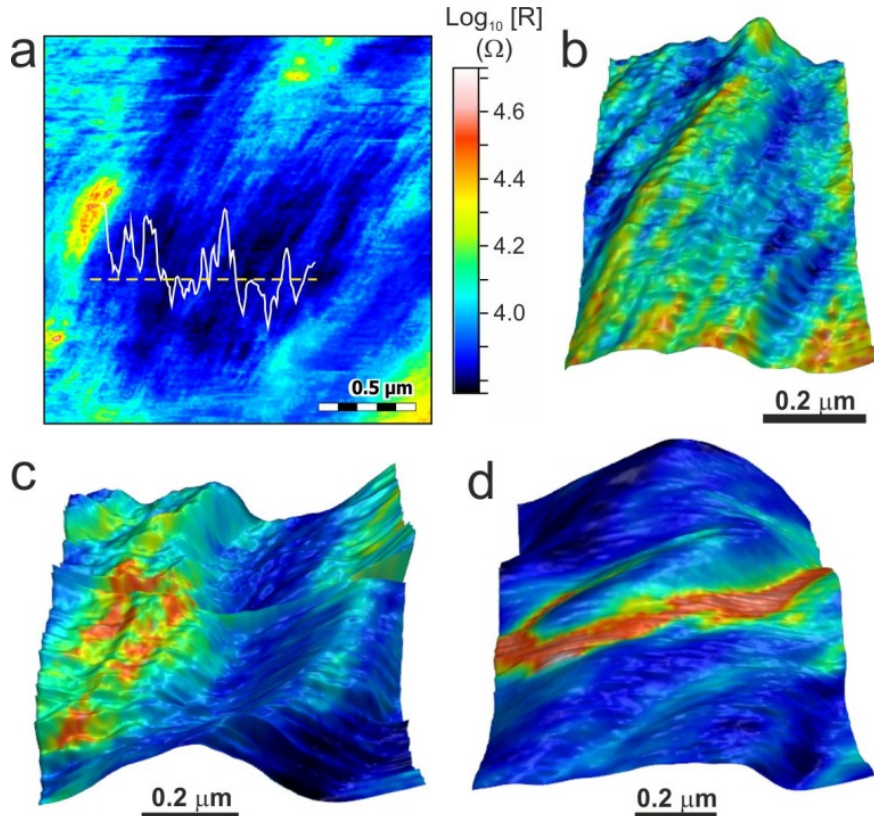

Fig. 6. (a) High resolution resistance map of G100. Inset: resistance profile along the indicated line. 3D topographic merged with resistance-coded color maps acquired in (b, c) G100 and (d) L100 samples. The maps were recorded applying $0.5 \mathrm{~V}$.

higher than $1 \mathrm{~T} \Omega$, which is the maximum detectable limit of the system. Interestingly, resistance maps obtained in G100 sample show, in addition to micrometric irregular areas with high resistance (hundreds of $k \Omega$ ), regions with parallel filament-like features of about tens of $k \Omega$ (Figs. $5 c$ and 6a). The resistive filaments, about one hundred nanometers in width and up to few microns in length, have similar dimensions to topographic filamentlike structures observed by AFM (Figs. $1 c$ and $2 b$ ). In order to correlate the resistance features with the surface morphology, three-dimensional high resolution topographic maps are coded with their corresponding resistance color (Fig. 6b). As observed, filamentary structures mostly show higher resistance at their topmost sites (crests) than in the trench between filaments. The measured difference in resistance is about $7 \%$. It should be noted that this difference in resistance is not provoked by inaccuracy in SPM topographic measurement, since our calculations from SPM deflection data point to around $1 \%$ of resistance deviation. Similarly, highly-resistive irregular regions present greater roughness than lower-resistive ones (Fig. 6c). Therefore, it can be pointed out that higher resistance regions are those which exhibit large density of structural defects. Besides, filament-like structures would be formed by somewhat self-organization of structural line defects, leading to the creation of resistive-conductive paths which promote percolation and charge transport along large distances, contributing to the decrease of macroscopic resistance. Conversely, L100 sample, which does not exhibit the formation of significant structural defects (Figs. 1d and 2c), reveals no formation of parallel conductive-resistive filament-like paths but randomly distributed irregular regions with different resistance values (Fig. 5c). Moreover, 3D topography-resistance maps show that highly resistive areas are indeed not correlated with the morphology at all, since they do not exhibit different morphology as compared to their surroundings (Fig. $6 \mathrm{~d}$ ). Then, it can be pointed out that these high resistance regions correspond to $\mathrm{GO}$ areas with high density of $\mathrm{sp}^{3}$ bonded functional groups. In consequence, the higher the oxidation degree, the larger resistance is measured. Due to the larger content of oxygen functionalities and their random distribution, poor percolation and number of conductive paths are created, leading to higher macroscopic resistance than in G100 sample. These results clearly indicate that laser-induced structural defects and chemical 
alterations play a multifaceted role in the macroscopic conductivity of processed GO materials.

\section{Conclusions}

Ultraviolet laser irradiation of flexible, free standing graphene oxide membrane in ammonia-rich gas and liquid environments was carried out. The samples submitted to analogous laser treatment exhibit remarkably different morphological and compositional properties depending on the nature of the surrounding medium. Thus, samples irradiated in gaseous ambient undergo a significant deoxygenation process, a slight incorporation of nitrogen species into the reduced graphene oxide structure and a large morphological modification. Conversely, the samples processed in liquid environment reveal a rather low reduction process but formation of significant amount of nitrogen functionalities, mainly in form of amine moieties, accompanied by slight morphological alteration. Though photochemical interactions play an important role in the deoxygenation development of $\mathrm{GO}$, numerical simulations point to a major influence of photothermal processes in the compositional change of the irradiated material, as well as the formation of structural defects in the graphene backbone. Resistance measurements evidence a direct influence of the composition and surface morphology configuration in the macroscopic conductivity of the processed material. The developed laser direct write method is facile, versatile, eco-friendly and easy to be implemented at industrial processes for the development of flexible devices, based on N-doped rGO materials.

\section{Acknowledgements}

The authors acknowledge the financial support of the Spanish Ministry of Economy and Competitiveness under the project ENE2014-56109-C3-3-R, in addition to the Romanian National Authority for Scientific Research and Innovation, CNCS UEFISCDI, under the Grants PN-II-ID-PCE-2012-4-0292 and PNII-RU-TE-2014-4-1194. ICMAB acknowledges financial support from the Spanish Ministry of Economy and Competitiveness, through the "Severo Ochoa" Programme for Centres of Excellence in R\&D (SEV-2015-0496).

\section{Notes and references}

1 A. C. Ferrari, F. Bonaccorso, V. Fal'ko, K. S. Novoselov, S. Roche, P. Boggild, S. Borini, F. H. L. Koppens, V. Palermo, N. Pugno, et al., Nanoscale, 2015, 7, 4598-4810.

2 S. Borini, R. White, D. Wei, M. Astley, S. Haque, E. Spigone, N. Harris, J. Kivioja and T. Ryhanen, Acs Nano, 2013, 7, 11166-11173.

3 H.-C. Hsu, I. Shown, H.-Y. Wei, Y.-C. Chang, H.-Y. Du, Y.-G. Lin, C.-A. Tseng, C.-H. Wang, L.-C. Chen, Y.-C. Lin and K.-H. Chen, Nanoscale, 2013, 5, 262-268.

4 K. Krishnamoorthy, R. Mohan and S. J. Kim, Applied Physics Letters, 2011, 98, 244101.
5 D. R. Dreyer, S. Park, C. W. Bielawski and R. S. Ruoff, Chemical Society Reviews, 2010, 39, 228-240.

6 T. Musso, P. V. Kumar, A. S. Foster and J. C. Grossman, Acs Nano, 2014, 8, 11432-11439.

7 S. E. Yalcin, C. Galande, R. Kappera, H. Yamaguchi, U. Martinez, K. A. Velizhanin, S. K. Doorn, A. M. Dattelbaum, M. Chhowalla, P. M. Ajayan, G. Gupta and A. D. Mohite, Acs Nano, 2015, 9, 2981-2988.

8 X. Wang, X. Li, L. Zhang, Y. Yoon, P. K. Weber, H. Wang, J. Guo and H. Dai, Science, 2009, 324, 768-771.

9 G. Venugopal, K. Krishnamoorthy, R. Mohan and S.-J. Kim, Materials Chemistry and Physics, 2012, 132, 29-33.

10 A. Iwase, Y. H. Ng, Y. Ishiguro, A. Kudo and R. Amal, Journal of the American Chemical Society, 2011, 133, 11054-11057.

11 T.-F. Yeh, J.-M. Syu, C. Cheng, T.-H. Chang and H. Teng, Advanced Functional Materials, 2010, 20, 2255-2262.

12 T. Schiros, D. Nordlund, L. Palova, D. Prezzi, L. Zhao, K. S. Kim, U. Wurstbauer, C. Gutierrez, D. Delongchamp, C. Jaye, D. Fischer, H. Ogasawara, L. G. M. Pettersson, D. R. Reichman, P. Kim, M. S. Hybertsen and A. N. Pasupathy, Nano Letters, 2012, 12, 4025-4031.

13 T.-F. Yeh, C.-Y. Teng, S.-J. Chen and H. Teng, Advanced Materials, 2014, 26, 3297-3303.

14 S. Seo, Y. Yoon, J. Lee, Y. Park and H. Lee, Acs Nano, 2013, 7, 3607-3615.

15 J. O. Hwang, J. S. Park, D. S. Choi, J. Y. Kim, S. H. Lee, K. E. Lee, Y.-H. Kim, M. H. Song, S. Yoo and S. O. Kim, Acs Nano, 2012, 6, 159-167.

16 H. Nolan, B. Mendoza-Sanchez, N. A. Kumar, N. McEvoy, S. O'Brien, V. Nicolosi and G. S. Duesberg, Physical Chemistry Chemical Physics, 2014, 16, 2280-2284.

17 T.-F. Yeh, S.-J. Chen, C.-S. Yeh and H. Teng, Journal of Physical Chemistry C, 2013, 117, 6516-6524.

18 S. Pei and H.-M. Cheng, Carbon, 2012, 50, 3210-3228.

19 R. Trusovas, G. Raciukaitis, G. Niaura, J. Barkauskas, G. Valušis and R. Pauliukaite. Advanced Optical Materials 2016, 4, 37-65.

20 W. Gao, N. Singh, L. Song, Z. Liu, A. L. M. Reddy, L. Ci, R. Vajtai, Q. Zhang, B. Wei and P. M. Ajayan, Nature Nanotechnology, 2011, 6, 496-500.

21 L. Guo, Y.-L. Zhang, D.-D. Han, H.-B. Jiang, D. Wang, X.-B. Li, H. Xia, J. Feng, Q.-D. Chen and H.-B. Sun, Advanced Optical Materials, 2014, 2, 120-125.

22 A. Pérez del Pino, E. György, C. Logofatu, J. Puigmartí-Luis and W. Gao. Carbon 2015, 93, 373-383.

23 A. Pérez del Pino, A. Datcu and E. György. Ceramics International 2016, 42, 7278-7283.

24 D. Bäuerle. Laser Processing and Chemistry (2000, Berlin: Springer).

25 D. C. Marcano, D. V. Kosynkin, J. M. Berlin, A. Sinitskii, Z. Sun, A. Slesarev, L. B. Alemany, W. Lu and J. M. Tour, Acs Nano, 2010, 4, 4806-4814.

26 D. Konios, M. M. Stylianakis, E. Stratakis and E. Kymakis, Journal of Colloid and Interface Science, 2014, 430, 108-112.

27 A. Perez del Pino, E. Gyoergy, L. Cabana, B. Ballesteros and G. Tobias, Journal of Applied Physics, 2014, 115, 093501.

28 J. C. Meyer, C. Kisielowski, R. Erni, M. D. Rossell, M. F. Crommie and A. Zettl, Nano Letters, 2008, 8, 3582-3586.

29 L. D. Carr and M. T. Lusk, Nature Nanotechnology, 2010, 5, 316-317.

30 Z. Qin, M. Taylor, M. Hwang, K. Bertoldi and M. J. Buehler, Nano Letters, 2014, 14, 6520-6525.

31 A. M. Alsayed, M. F. Islam, J. Zhang, P. J. Collings and A. G. Yodh, Science, 2005, 309, 1207-1210.

32 T. T. D. Huynh, M. Vayer, A. Sauldubois, A. Petit and N. Semmar, Applied Physics Letters, 2015, 107, 193105.

33 A. Bagri, C. Mattevi, M. Acik, Y. J. Chabal, M. Chhowalla and V. B. Shenoy, Nature Chemistry, 2010, 2, 581-587.

34 M. Acik, C. Mattevi, C. Gong, G. Lee, K. Cho, M. Chhowalla and Y. J. Chabal, Acs Nano, 2010, 4, 5861-5868. 
35 Y. Matsumoto, M. Koinuma, S. Ida, S. Hayami, T. Taniguchi, K. Hatakeyama, H. Tateishi, Y. Watanabe and S. Amano, Journal of Physical Chemistry C, 2011, 115, 19280-19286.

36 C. Gomez-Navarro, J. C. Meyer, R. S. Sundaram, A. Chuvilin, S. Kurasch, M. Burghard, K. Kern and U. Kaiser, Nano Letters, 2010, 10, 1144-1148.

37 G. Eda, G. Fanchini and M. Chhowalla, Nature Nanotechnology, 2008, 3, 270-274.

38 Z. Wei, D. Wang, S. Kim, S.-Y. Kim, Y. Hu, M. K. Yakes, A. R. Laracuente, Z. Dai, S. R. Marder, C. Berger, W. P. King, W. A. de Heer, P. E. Sheehan and E. Riedo, Science, 2010, 328, 1373-1376.

39 R. Larciprete, S. Fabris, T. Sun, P. Lacovig, A. Baraldi and S. Lizzit, Journal of the American Chemical Society, 2011, 133, 17315-17321.

40 S. Moussa, A. R. Siamaki, B. F. Gupton and M. S. El-Shall, Acs Catalysis, 2012, 2, 145-154.

41 B. J. Schultz, R. V. Dennis, J. P. Aldinger, C. Jaye, X. Wang, D. A. Fischer, A. N. Cartwright and S. Banerjee, Rsc Advances, 2014, 4, 634-644.

42 C. Gomez-Navarro, R. T. Weitz, A. M. Bittner, M. Scolari, A Mews, M. Burghard and K. Kern, Nano Letters, 2007, 7, 34993503.

43 A. H. Castro Neto, F. Guinea, N. M. R. Peres, K. S. Novoselov and A. K. Geim, Reviews of Modern Physics, 2009, 81, 109162. 